Commun. math. Phys. I3, 335-344 (1969)

\title{
Critical Temperature Bounds of Quantum Lattice Gases
}

\author{
W. Greenberg \\ Department of Mathematics, Indiana University \\ Bloomington, Indiana
}

Received March 14, 1969

\begin{abstract}
We prove rigorous critical temperature upper bounds of quantum lattices in the infinite volume limit and with many-body potentials which conserve the number of particles. The results are obtained from the analyticity properties of the reduced correlation functionals. As an example the isotropic Heisenberg model is considered. The method also extends previous results on the analyticity and the critical temperature of a classical lattice.
\end{abstract}

\section{Introduction}

In recent papers $[1,3]$, it has been proved that the reduced correlation functionals of a quantum lattice gas in the infinite volume limit are analytic in fugacity $z$ in a region of the $\beta-z$ plane corresponding to high temperatures. The method depends upon estimates on the kernels of a set of integral equations for the finite volume correlation functionals. Here we consider potentials which conserve particle number, and we extend the region of analyticity sufficiently to obtain an upper bound on the critical temperature of the lattice. In addition, for particular potentials we provide a means of selecting contributions to the kernels which are significant, and of estimating the remainder. In the last sections, the results are applied to the isotropic Heisenberg model and to classical lattices. Enlarged regions of analyticity and improved critical temperature bounds are obtained for both the classical $[2,4]$ and the quantum $[1,5]$ potentials.

\section{Notation}

The states of a $\nu$-dimensional lattice $\mathbb{Z}^{v}$ are given by subsets $X \subset \mathbb{Z}^{v}$. If we associate with each lattice point $x$ a two-dimensional vector space $\mathscr{H}_{x}$ generated by the creation and annihilation operators $a_{x}^{+}, a_{x}$, and with each finite subset the tensor product $\mathscr{H}=\bigotimes_{x \in \Lambda} \mathscr{H}_{x}$, then the vector $|X\rangle=a^{+}(X)|\emptyset\rangle$ in $\mathscr{H}, X \subset \Lambda$, corresponds to a state of the lattice with sites $y \in X$ occupied and $y \in \Lambda-X$ unoccupied. Let $\mathfrak{A}(\Lambda)$ denote the bounded operators on $\mathscr{H}_{\Lambda}$. 
Interactions of the lattice are given by a sequence $\left\{\varphi^{n}\right\}$ of potentials, where $\varphi^{n}\left(x_{1}, \ldots, x_{n}\right)$ is an $n$-body potential which is a Hermitian, translation-covariant, bounded operator on $\mathscr{H}_{x_{1}} \cup \ldots \cup x_{n}$. We shall assume that $\left\{\varphi^{n}\right\}$ commutes with the number of particles operator: $\left[\varphi^{n}\left(x_{1}, \ldots, x_{n}\right), N\right]=0$ for all $n$, where $N=\sum_{x \in Z^{\nu}} a_{x}^{+} a_{x}$. In addition, we

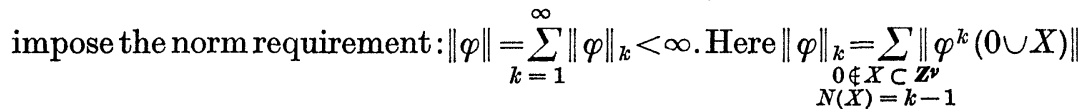
and $\left\|\varphi^{k}(Y)\right\|$ is the operator norm of $\varphi^{k}(Y)$. With this norm the potentials form a Banach space $B$. Translation covariance and commutation with $N$ implies $\beta \varphi^{1}(x)=-\ln z a_{x}^{+} a_{x}$ (plus a multiple of the identity, which corresponds to an irrelevant rescaling of the energy), and this serves to define the fugacity $z$. The energy operator is $U_{\varphi}(\Lambda)=\sum_{X \subset \Lambda} \varphi(X)$.

The correlation functional for a lattice of finite volume $\Lambda$ is defined by $\varrho_{\Lambda}^{\varphi}(X, Y)=Z_{\Lambda}^{-1} \operatorname{Tr}\left\{e^{-\beta U_{\varphi}(\Lambda)} a^{+}(X) a(Y)\right\}$. Then $\varrho_{\Lambda} \in \mathscr{L}^{\infty}$, the Banach space of bounded sequences, and satisfies a generalized Kirkwood-Salzburg integral equation [3]:

Theorem 2.1. $\varrho_{\Lambda}=\left(I-K_{\Lambda}\right)^{-1} \alpha$, where $\alpha \in \mathscr{L}^{\infty}$ and $K_{\Lambda} \in \operatorname{Hom}\left(\mathscr{L}^{\infty}\right)$, the algebra of bounded operators on $\mathscr{L}^{\infty}$. The matrix elements of $K_{\Lambda}$ are given by

$$
K_{\Lambda}\left(X, Y ; P, Y^{\prime} \cup R\right)=\left\{\begin{aligned}
\sum_{\substack{V \subset R \\
R \cap\left(y_{1} \cup X\right) \subset V}}(-1)^{N(V)}\langle P-V| e^{\beta U(\Lambda)} a_{y_{1}} e^{-\beta U(\Lambda)} & |X \cup(R-V)\rangle \\
& \text { if } Y \neq \emptyset,\left(X \cup y_{1}\right) \cap R \subset P \\
\sum_{\substack{V R \cap\left(P-X^{\prime}\right) \\
x_{1} \cap P \subset V}}^{(-1)^{N(\Lambda)}\left\langle P-X^{\prime}-V\right| e^{-\beta U(\Lambda)} a_{x_{1}}^{+}} & \text {if } Y=\emptyset, x_{1} \cap P \subset R, X^{\prime} \subset P
\end{aligned}\right.
$$

and zero otherwise, for $y_{1} \in Y, Y^{\prime}=Y-y_{1}, x_{1} \in X, X^{\prime}=X-x_{1}$, and

$$
\alpha(X, Y)=\left\{\begin{array}{l}
1 \text { if } X \cup Y=\emptyset \\
0 \text { otherwiese. }
\end{array}\right.
$$

\section{General Quantum Potentials}

A subset $B_{c}, c \in \mathbb{R}$, of the Banach space $B$ is given by those potentials in $B$ which satisfy: $\Gamma(\varphi)=(2 / \pi)^{1 / 4} \cdot \sum_{n=1}^{\infty} \sum_{k_{1}=2}^{\infty} \cdots \sum_{k_{n}=2}^{\infty}\|\varphi\|_{k_{1}} \ldots\|\varphi\|_{k_{n}} \sum_{r=0}^{Q(n)}$

$$
\cdot \sum_{j=0}^{r}\left(\begin{array}{l}
Q(n) \\
r
\end{array}\right)\left(\begin{array}{l}
r \\
j
\end{array}\right) \frac{2^{n+\frac{1}{2}(Q(n)-j)}}{\left(Q(n)-j+\frac{2}{\pi} \delta(Q(n)=j)\right)^{1 / 4}} \frac{1}{n !} \cdot \prod_{s=1}^{n-1} Q(s)<c
$$

for $Q(s)=\sum_{i=1}^{s}\left(k_{i}-1\right)+1$ and $\delta(A=B)= \begin{cases}1, & A=B \\ 0, & \text { otherwise } .\end{cases}$ 
Theorem 3.1. If $\beta \varphi \in B_{c}, c<z^{-1}$, then in the limit $\Lambda \rightarrow \infty, K_{\Lambda} \rightarrow K$ $\in \operatorname{Hom}\left(\mathscr{L}^{\infty}\right)$ in the sense that the adjoints converge weakly in $\mathscr{L}^{1}$. Thus $\varrho_{\Lambda}(X, Y) \rightarrow \varrho(X, Y)$ pointwise, and the functions $\varrho(X, Y)$, the infinite volume correlation functionals, are analytic in fugacity $z$.

The proof of 3.1, as well as much of what is to follow, depends upon an estimate of the kernels $K$ which is proved in [3]. Expand $K_{A}$ in multicommutators to obtain $K_{\Lambda}^{n}, K_{\Lambda}=\sum_{n=0}^{\infty} \frac{1}{n !} K_{\Lambda}^{n}$.

Theorem 3.2.

$$
\begin{gathered}
\sum_{\substack{P, R \subset A \\
R \cap Y^{\prime}=\emptyset}}\left\|K_{\Lambda}^{n}\left(X, Y ; P, Y^{\prime} \cup R\right)\right\| \leqq \sum_{y_{2} \in S_{1}} \cdots \sum_{y_{n} \in S_{n-1}} \sum_{\substack{Y_{1} \subset A \\
Y_{1} \cap y=\emptyset}} \cdots \sum_{\substack{Y_{n} \subset A \\
Y_{n} \cap y_{n}=\emptyset}} \\
\quad \sum_{P, R \subset S_{n}} \sum_{V \subset R \cap P} \beta^{n} \mid\langle P-V|\left[\varphi\left(y_{n} \cup Y_{n}\right), \ldots,\left[\varphi\left(Y_{1} \cup y\right), a_{y}\right] \ldots\right] \\
\cdot\left|\left(Y \cap S_{n}\right) \cup(R-V)\right\rangle \mid \text { where } S_{p}=Y_{p} \cup Y_{p-1} \cup \cdots \cup Y_{1} \cup y .
\end{gathered}
$$

Proof of 3.1. In [3], the Schwarz inequality is employed to sum over $P$. However, as $\varphi$ conserves particle number, $\left\langle P-V\left|\left[\varphi, a_{y}\right]^{(n)}\right|\left(X \cap S_{n}\right)\right.$ $\cup(R-V)\rangle=0$ unless $N(P-V)=N\left(\left(X \cap S_{n}\right) \cup(R-V)\right)-1$, since $\left[\varphi, a_{y}\right]^{(n)} \equiv H$ annihilates one particle. Therefore, there are not $2^{N\left(S_{n}\right)-N(V)}$ terms in the sum over $P$, but at most $\sup _{0 \leqq j \leqq N\left(S_{n}\right)-N(V)}\left(\begin{array}{c}N\left(S_{n}\right)-N(V) \\ j\end{array}\right)$. Use the inequality $\sup _{j}\left(\begin{array}{c}N\left(S_{n}\right)-N(V) \\ j\end{array}\right) \leqq(2 / \pi)^{1 / 2} \frac{2^{N\left(S_{n}\right)-N(V)}}{\left(N\left(S_{n}\right)-N(V)+\frac{2}{\pi} \delta\right)^{1 / 2}}$, where the $\delta$ indicates that the denominator is to be taken equal to $\sqrt{2 / \pi}$ if $N\left(S_{n}\right)-N(V)=0$. Then $\sum_{R \subset S_{n}} \sum_{V \subset R} \sum_{T \subset S_{n}-V}\left|\left\langle T|H|\left(X \cap S_{n}\right) \cup(R-V)\right\rangle\right|$

$$
\begin{aligned}
& \leqq \sum_{R \subset S_{n}} \sum_{V \subset R}\|H\|\left\{\sqrt{\frac{2}{\pi}} \frac{2^{N\left(S_{n}\right)-N(V)}}{\left(N\left(S_{n}\right)-N(V)+\frac{2}{\pi} \delta\right)^{1 / 2}}\right\}^{1 / 2} \\
& \left.\leqq \sum_{r=0}^{N\left(S_{n}\right)}\left(\begin{array}{c}
N\left(S_{n}\right) \\
r
\end{array}\right) \sum_{j=0}^{r} \begin{array}{l}
r \\
j
\end{array}\right)(2 / \pi)^{1 / 4} \frac{2^{\frac{1}{2}\left(N\left(S_{n}\right)-j\right)}}{\left(\left(N\left(S_{n}\right)-j+\frac{2}{\pi} \delta\right)^{1 / 4}\right.}\|H\| .
\end{aligned}
$$

From 3.2 and the inequalities $\left\|\left[\varphi, a_{y}\right]^{(n)}\right\| \leqq 2^{n}\|\varphi\|^{n}, N\left(S_{p}\right) \leqq Q(p)$, obtain $\left\|K_{\Lambda}^{n}\right\| \leqq(2 / \pi)^{1 / 4} 2^{n} \beta^{n} \sum_{k_{1}=2} \ldots \sum_{k_{n}=2}\|\varphi\|_{k_{1}} \ldots\|\varphi\|_{k_{n}} \sum_{r=0}^{Q(n)} \sum_{j=0}^{r}\left(\begin{array}{c}Q(n) \\ r\end{array}\right)$

$$
\cdot\left(\begin{array}{l}
r \\
j
\end{array}\right) \frac{2^{\frac{1}{2}(Q(n)-j)}}{\left(Q(n)-j+\frac{2}{\pi} \delta\right)^{1 / 4}} \underset{s=1}{n-1} Q(s) .
$$

To prove $\sup _{j}\left(\begin{array}{c}N \\ j\end{array}\right) \leqq \sqrt{2 / \pi} 2^{N}\left(N+\frac{2}{\pi} \delta\right)^{-1 / 2}$, assume $N$ odd and use the Stirling expansion: $n !=\sqrt{2 \pi / n} n^{n} e^{-n} e^{r(n)}$, where $r(n)$ is an 23 Commun.math. Phys., Vol. 13 
error term which satisfies $1 / 12 n-1 / 360 n^{3}<r(n)<1 / 12 n$ [6]. Then $\sup _{j}\left(\begin{array}{c}N \\ j\end{array}\right)=N ! /((N+1) / 2) !((N-1) / 2) ! \leqq \sqrt{2 / \pi} 2^{N} N^{-1 / 2} N^{N+1}(N-1)^{-N / 2}$ $\cdot(N+1)^{-1-N / 2} \exp \left(1 / 12 N-1 / 6(N+1)+1 / 45(N+1)^{3}-1 / 6(N-1)\right.$ $+1 / 45(N-1)^{3}$. However, $\frac{1}{12 N}-\frac{1}{6(N+1)}+\frac{1}{45(N+1)^{3}}-\frac{1}{6(N-1)}$ $+\frac{1}{45(N-1)^{3}}<0$ for $N>2$, and $N^{N+1}(N-1)^{-\frac{1}{2} N}(N+1)^{-1-\frac{1}{2} N}$ $=\left(\left(1-\frac{1}{N^{2}}\right)\left(1+\frac{1}{N}\right)^{2 / N}\right)^{-1}<1$ for $N>2$, since :

$$
\begin{aligned}
& \left(1-\frac{1}{N^{2}}\right)\left(1+\frac{1}{N}\right)^{2 / N}=\left(1-\frac{1}{N^{2}}\right)\left(1+\frac{2}{N} \cdot \frac{1}{N}+\frac{1}{2 ! N^{2}}\left(\frac{2}{N}\right)\left(\frac{2}{N}-1\right)\right. \\
& \left.\quad+\frac{1}{3 ! N^{3}}\left(\frac{2}{N}\right)\left(\frac{2}{N}-1\right)\left(\frac{2}{N}-2\right)+\cdots\right) \\
& \quad \geqq\left(1-\frac{1}{N^{2}}\right)\left(1+\frac{2}{N^{2}}-\frac{2}{N}\left(\frac{1}{2 !} \cdot \frac{1}{N^{2}}+\frac{3 !}{4 !} \cdot \frac{1}{N^{4}}+\frac{5 !}{6 !} \cdot \frac{1}{N^{6}}+\cdots\right)\right) \\
& =1+\frac{1}{N^{2}}-\frac{1}{N^{3}}-\frac{2}{N^{4}}+\frac{2}{N}\left(\left(\frac{1}{2}-\frac{1}{4}\right) \frac{1}{N^{4}}+\left(\frac{1}{4}-\frac{1}{6}\right) \frac{1}{N^{6}}+\cdots\right) \\
& \geqq 1+\frac{1}{N^{2}}-\frac{1}{N^{3}}-\frac{2}{N^{4}} \geqq 1 .
\end{aligned}
$$

If $K(X, Y ; P, R)$ is chosen to be the limit $\lim _{\Lambda \rightarrow \infty} K_{\Lambda}(X, Y ; P, R)$, then $\lim \sum_{P, R}\left\|K_{\Lambda}\left(X, Y ; P, R \cup Y^{\prime}\right)-K\left(X, Y ; P, R \cup Y^{\prime}\right)\right\|=0$ uniformly in fugacity. From this and the bound $\left\|K_{\Lambda}\right\|<$ constant $<1$, it follows that for any $\chi \in \mathscr{L}^{1}$ and $\xi \in \mathscr{L}^{\infty}, \chi\left(K_{\Lambda} \xi\right) \rightarrow \chi(K \xi)$. Then, for $\chi(X, Y)$ $=\delta(X, Y)$ and $\xi=\alpha$, the convergence of $\chi\left(\frac{1}{1-K_{\Lambda}} \xi\right) \rightarrow \chi\left(\frac{1}{1-K} \xi\right)$ uniformly in $z$ completes the proof of the theorem.

It is possible to extend the region of analyticity in the $\beta-z$ plane by employing hole-particle symmetry. Toward this end, define the holeparticle inversion $\mathscr{L}:\left(\mathscr{L}^{-1} B_{c}\right) \rightarrow B_{c}$ by

$$
(\mathscr{L} \varphi)(X)=(-1)^{N(X)} \sum_{Y \supset X} \operatorname{Tr}_{\mathscr{H}_{Y-X}} \varphi(Y)
$$

which may be interpreted as giving physical significance to unoccupied lattice sites (holes) rather than particles, and define the symmetry operator $s: \mathfrak{A}(\Lambda) \rightarrow \mathfrak{A}(\Lambda)$ by

$$
s(A)=\sum_{T \subset \Lambda}(-1)^{N(T)} \operatorname{Tr}_{\mathscr{H}_{\Lambda-T}} A .
$$

Note $\mathscr{L}^{2}=I, s^{2}=I$. These generalize to quantum lattices the holeparticle symmetry of a classical lattice dissussed in [2].

Lemma 3.3. $A \in \mathcal{U}(\Lambda) \Rightarrow \operatorname{Tr}_{\mathscr{H}_{\Lambda}} A=\operatorname{Tr}_{\mathscr{H}_{\Lambda}} s(A)$.

Proof. $\operatorname{Tr} s(A)=\sum_{T \subset A} \sum_{V \subset A}(-1)^{N}(V) \sum_{W \subset A-V}\langle(T \cap V) \cup W|A|(T \cap V) \cup W\rangle$ $=\sum_{T \subset \Lambda} \sum_{\substack{W_{1} \subset \Lambda \\ W_{1} \cap T=\emptyset}} \sum_{W_{2} \subset T} \sum_{\substack{V_{2} \subset T \\ V_{2} \cap W_{2}=\emptyset}} \sum_{\substack{V_{1} \subset \Lambda-T \\ V_{1} \cap W_{1}=\emptyset}}(-1)^{N\left(V_{1}\right)-N\left(V_{2}\right)}\left\langle V_{2} \cup W_{1} \cup W_{2}|A| V_{2}\right.$ $\left.\cup W_{1} \cup W_{2}\right\rangle$, 
where we have written $V_{2}=V \cap T, V_{1}=V-V_{2}, W_{2}=W \cap T$, and

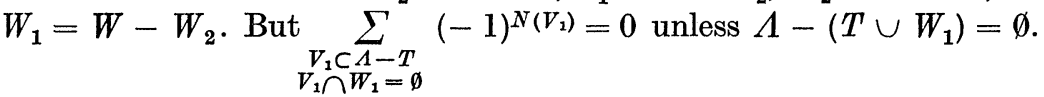

Therefore $T=\Lambda-W_{1}, V_{1}=\emptyset, \quad$ and

$\operatorname{Tr} s(A)$

$$
\begin{aligned}
& =\sum_{W_{1} \subset A} \sum_{\substack{W_{2} \subset \Lambda \\
W_{1} \cap W_{2}=\emptyset}} \sum_{\substack{V_{2} \subset \Lambda \\
V_{2} \cap\left(W_{1} \cup W_{2}\right)=\emptyset}}(-1)^{N\left(V_{2}\right)}\left\langle V_{2} \cup W_{1} \cup W_{2}|A| V_{2} \cup W_{1} \cup W_{2}\right\rangle \\
& =\sum_{R \subset \Lambda}\langle R|A| R\rangle D(R),
\end{aligned}
$$

where $D(R)$ is the number of ways of disjointly choosing $V_{2}, W_{1}, W_{2}$, so that $V_{2} \cup W_{1} \cup W_{2}=R$, weighted by $(-1)^{N\left(V_{2}\right)}$. Thus, $D(R)=\sum_{n=0}^{N(R)}$ $\cdot\left(\begin{array}{c}N(R) \\ n\end{array}\right) \sum_{j=0}^{N(R)-n}\left(\begin{array}{c}N(R)-n \\ j\end{array}\right)(-1)^{n}=\sum_{n=0}^{N(R)}\left(\begin{array}{c}N(R) \\ n\end{array}\right)(-1)^{n}(1+1)^{N(R)-n}$ $=(1+1-1)^{N(R)}=1$ and $\operatorname{Tr} s(A)=\sum_{R \subset A}\langle R|A| R\rangle=\operatorname{Tr} A$.

Lemma 3.4. $A, B \in \mathfrak{A}(\Lambda) \Rightarrow s(A B)=s(B) s(A)$.

Proof. The case $N(\Lambda)=1$ is trivial. Assume $A, B \in \mathfrak{A}(\Lambda)$ and observe $s$ is linear on $\mathfrak{A}(x)$. Then $s(A B)=\sum_{T \subset \Lambda}(-1)^{N(T)} \operatorname{Tr}_{\mathscr{H} \Lambda-T}\left(\bigotimes_{x \in \Lambda} A_{x} B_{x}\right)$ $=\bigotimes_{x \in \Lambda}\left(\sum_{y \subset x}(-1)^{N(y)} \operatorname{Tr}_{\mathscr{H}_{x-y}} A_{x} B_{x}\right)=\bigotimes_{x \in \Lambda} s\left(A_{x} B_{x}\right)=\bigotimes_{x \in \Lambda} s\left(B_{x}\right) s\left(A_{x}\right)$ $=s\left(\bigotimes_{x \in \Lambda} B_{x}\right) s\left(\bigotimes_{x \in \Lambda} A_{x}\right)=s(B) s(A)$.

Lemma 3.5. $s\left(U_{\varphi}(\Lambda)\right)=U_{\mathscr{L}_{\varphi}}(\Lambda)+N(\Lambda) E_{0}+S(\Lambda)$, where $E_{0}=\sum_{0 \in X} \operatorname{Tr}_{\mathscr{H}_{X}} \varphi(X) / N(X)$ and $S(\Lambda)$ is a boundary term with the property: $\operatorname{Lim}_{\Lambda \rightarrow \infty}\|S(\Lambda)\| / N(\Lambda)=0$.

Proof. From the definition of $s$ and $\mathscr{L}$ : $s\left(U_{\varphi}(\Lambda)\right)$

$=\sum_{X \subset \Lambda} \sum_{T \subset X}(-1)^{N(T)} \operatorname{Tr}_{\mathscr{H}_{X-T}} \varphi(X)=\sum_{T \subset \Lambda}\left\{(-1)^{N(T)} \sum_{\substack{X \subset \Lambda \\ X \supset T}} \operatorname{Tr}_{\mathscr{H}_{X-T}} \varphi(X)\right\}$ $=\sum_{\substack{T \subset \Lambda \\ T \neq \emptyset}}(\mathscr{L} \varphi)(T)+(\mathscr{L} \varphi)(\emptyset)+S_{1}(\Lambda)$

where

$$
S_{1}(\Lambda)=\sum_{T \subset \Lambda}(-1)^{N(T)} \sum_{\substack{x \supset T \\ X \cap \Lambda \neq X}} \operatorname{Tr}_{\mathscr{H}_{X-T}} \varphi(X)
$$

is a boundary term. Then compute:

$$
\begin{aligned}
(\mathscr{L} \varphi)(\emptyset) & =\sum_{Y} \operatorname{Tr}_{\mathscr{H}_{Y}} \varphi(Y)=\sum_{n=1}^{\infty} \sum_{y \in \mathbb{Z}^{v}} \sum_{\substack{y \notin Y \subset \mathbb{Z}^{v} \\
N(Y)=n-1}} \operatorname{Tr}_{\mathscr{H}_{y \cup Y}} \varphi(y \cup Y) / n \\
& =\sum_{n=1}^{\infty} N(\Lambda) \sum_{\substack{Y \oplus 0 \\
N(Y)}} \operatorname{Tr}_{\mathcal{H}^{*}} \operatorname{Tr}_{\mathscr{H}_{0} \cup Y} \varphi(0 \cup Y) / n+S_{2}(\Lambda) .
\end{aligned}
$$


Corollary 3. 6. The pressure $P_{\Lambda}(\varphi)=(1 / N(\Lambda)) \log \operatorname{Tr}_{\mathscr{H}_{\Lambda}} e^{-\beta U_{\varphi}(\Lambda)}$ satisfies $P_{\Lambda}(\varphi)=P_{\Lambda}(\mathscr{L} \varphi)-\beta E_{0}+S_{0}(\Lambda)$ with $\operatorname{Lim}_{\Lambda \rightarrow \infty} S_{0}(\Lambda)=0$.

Proof. Use the bounds due to van Kampen [7]:

$P_{\Lambda}(\varphi)-\beta \sigma / N(\Lambda) \leqq(1 / N(\Lambda)) \log \operatorname{Tr}_{\mathscr{H}{ }_{\Lambda}} e^{-\beta\left(U_{\varphi}(\Lambda)+S\right)} \leqq P_{\Lambda}(\varphi)-\beta \tau / N(\Lambda)$, where $\sigma$ and $\tau$ are the maximum and minimum eigenvalues, respectively, of $S$.

Theorem 3.7. If $\beta \mathscr{L} \varphi$ and $\beta \varphi$ both satisfy the hypothesis of Theorem $\begin{aligned} 3.1, \text { then } \varrho^{\varphi}(X, Y)= & (-1)^{N(X)+N(Y)} \sum_{T \subset X \cap Y}(-1)^{N(T)} \varrho^{\mathscr{L} \varphi} \\ & \cdot(X-X \cap Y+T, Y-X \cap Y+T) .\end{aligned}$

Proof. For a lattice of finite volume:

$$
\begin{aligned}
\varrho_{\Lambda}(X, Y)= & Z_{\Lambda}^{\varphi-1} \operatorname{Tr}\left\{s\left(a(Y) e^{-\beta U_{\varphi}} a^{+}(X)\right)\right\}=Z_{\Lambda}^{\varphi-1}(-1)^{N(X)+N(Y)} \\
& \cdot \operatorname{Tr}\left\{a^{+}(X) e^{-\beta s\left(U_{\varphi}\right)} a(Y)\right\} \\
= & (-1)^{N(X)+N(Y)} Z_{\Lambda}^{\varphi-1} \sum_{S \supset X \cup Y}\left\langle S-X\left|e^{-\beta s\left(U_{\varphi}\right)}\right| S-Y\right\rangle
\end{aligned}
$$

since all partial traces of $a(X)$ on $\mathscr{H}_{T}$ vanish for $T \subset X$ except $\operatorname{Tr}_{\mathscr{H} \emptyset} a(X)$ $=(-1)^{N(X)} a(X)$. Let $X=\hat{X}+X \cap Y, Y=\hat{Y}+X \cap Y$, and compute from Lemma 3.5:

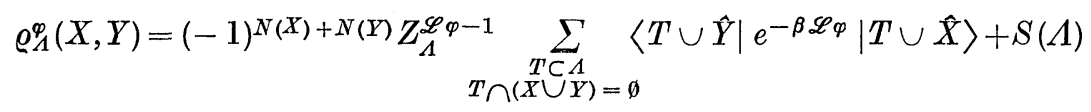

with $\operatorname{Lim}_{\Lambda \rightarrow \infty} S(\Lambda)=0$. Then from the identity

letting

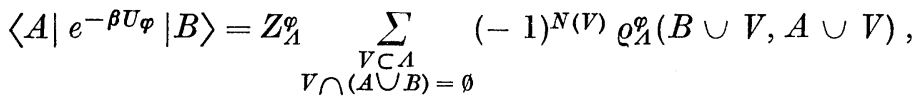

$$
\begin{aligned}
& T \cup V=W, W-(X \cap Y) \cap W=V^{\prime}, \\
& \varrho_{\Lambda}^{\varphi}(X, Y)=(-1)^{N(X)+N(Y)} \sum_{\substack{W \subset A \\
V^{\prime} \subset W-(X \cap Y) \cap W \\
W \cap(\hat{X} \cup \hat{Y})=\emptyset}}(-1)^{N(X \cap Y \cap W)} \\
& \cdot(-1)^{N\left(V^{\prime}\right)} \varrho_{\Lambda}^{\mathscr{L} \varphi}(\hat{X} \cup W, \hat{Y} \cup W)+S(\Lambda) .
\end{aligned}
$$

However, the sum $\sum_{V^{\prime} \subset W-(X \cap Y) \cap W}(-1)^{N\left(V^{\prime}\right)}$ vanishes unless $W-(X \cap Y) \cap W=\emptyset$, thus $W \subset X \cap Y$, and the theorem follows from the finiteness of the sum over $T \subset X \cap Y$ for finite $X, Y$ as $A \rightarrow \infty$.

Define the transformed fugacity $\mathscr{L} z$ by $(\mathscr{L} \varphi)^{1}(x)=\mu I-\beta^{-1}$ - $\log (\mathscr{L} z) a_{x}^{+} a_{x}, \mu, \mathscr{L} z \in \mathbb{R}$.

Corollary 3.8. If $\beta \mathscr{L}_{\varphi} \in B_{c}, c<(\mathscr{L} z)^{-1}$, but $\beta \varphi \notin B_{c}$, then 3.6 provides an analytic continuation of the components $\varrho^{\varphi}(X, Y)$ of $\varrho^{\varphi}$. 
Theorem. 3.9. Suppose $\varphi \in B_{c}, c<\infty$, and define $\Gamma(\varphi)$ as before Theorem 3.1. Then the critical temperature $T_{0}$ of an infinite volume lattice with potential $\varphi$ is bounded by $T_{0} \leqq\left(k \beta_{1}\right)^{-1}$ for $\beta_{1}$ the solution of the simultaneous equations: $z \Gamma(\beta \varphi)=1, \mathscr{L} z \Gamma(\beta \mathscr{L} \varphi)=1$.

Theorem 3.9 follows directly from the observation that $\Gamma(\beta \varphi)$ is a monotonically decreasing function of $\beta$.

A case of special interest is the general two-body potential, $\varphi^{i}=0$, $i>2$.

Corollary 3.10. If $\varphi \in B_{c}, c<\infty$, and $\varphi^{i}=0, i>2$, then the critical temperature $T_{0}$ of the infinite volume lattice satisfies $T_{\mathbf{0}} \leqq 31\|\varphi\| / k$.

Proof. Compute $\mathscr{L} \varphi \cdot(\mathscr{L} \varphi)^{i}=0, i>2$, and $\left(\mathscr{L}_{\varphi)^{2}}=\varphi^{2} \cdot(\mathscr{L} \varphi)^{1}(0)\right.$ $=z^{-1} \exp \left\{-\beta \sum_{x \neq 0} \operatorname{Tr}_{\mathscr{H}_{x}} \varphi(0 \cup x)\right\}$, and since $\left\|\sum_{x \neq 0} \operatorname{Tr}_{\mathscr{H}_{x}} \varphi(0 \cup x)\right\| \leqq 2\|\varphi\|$, $z^{-1} e^{-4 \beta\|\varphi\|} \leqq \mathscr{L} z \leqq z^{-1} e^{4 \beta\|\varphi\|}$. As $\varrho^{\mathscr{L} \varphi}$ is analytic for $\mathscr{L} z<1 / \Gamma(\beta \varphi)$, $\varrho^{\varphi}$ is analytic for $z^{-1} e^{4 \beta\|\varphi\|}<1 / \Gamma(\beta \varphi)$, and the critical temperature is determined by the solution $\beta_{1}$ of $z=1 / \Gamma(\beta \varphi), z^{-1}=e^{-4 \beta\|\varphi\|} / \Gamma(\beta \varphi)$.

For a two-body potential, $k_{i}=2, Q(s)=s+1$, and $\Gamma(\beta \varphi)=(2 / \pi)^{1 / 4}$ $\cdot \sum_{n=1}^{\infty}(2 \beta\|\varphi\|)^{n} \sum_{r=0}^{n+1} \sum_{j=0}^{r}\left(\begin{array}{c}n+1 \\ r\end{array}\right)\left(\begin{array}{l}r \\ j\end{array}\right) \frac{2^{\frac{1}{2}(n+1-j)}}{\left(n+1-j+\frac{2}{\pi} \delta\right)^{1 / 4}}$. The critical temperature bound is the solution of $\sum_{n=1}^{\infty} c_{n} x^{n}=\exp (-x / 2 / \overline{2})$ with $x=2 \sqrt{2} \beta_{1}\|\varphi\|$ and $c_{n}=4 \sqrt{\frac{8}{\pi}} \sum_{r=0}^{n+1} \sum_{j=0}^{r}\left(\begin{array}{c}n+1 \\ r\end{array}\right)\left(\begin{array}{l}r \\ j\end{array}\right) 2^{-\frac{1}{2} j}\left(n+1-j+\frac{2}{\pi} \delta\right)^{-1 / 4}$. Calculating the first several $c_{n}$ and estimating the remainder by $c_{n} \leqq\left(\frac{4}{\pi}\right)^{1 / 4} \sum_{r=0}^{n+1} \sum_{j=0}^{r}$ $\cdot\left(\begin{array}{c}n+1 \\ r\end{array}\right)\left(\begin{array}{l}r \\ j\end{array}\right) 2^{-\frac{1}{2} j}=\left(\frac{4}{\pi}\right)^{1 / 4}\left(2+\sqrt{\frac{1}{2}}\right)^{n+1}$ gives $x=0.09$.

\section{The Heisenberg Model}

The estimates of $\left\|K_{\Lambda}^{n}\right\|$ in Theorem 3.2, along with the proof of 3.1, provide a method of obtaining an improved upper bound on the critical temperature for some potentials. Namely, the multiple-commutators and their matrix elements cccurring in 3.2 can be explicitly evaluated for the first few partial kernels $K_{\Lambda}^{n}$, thereby obtaining better bounds on $\left\|K_{\Lambda}^{n}\right\|, n=1,2, \ldots, N$, and then the remaining partial kernels can be estimated as in 3.1.

As an example, consider the isotropic Heisenberg model: $\varphi^{2}(x, y)$ $=V(x, y) a_{x}^{+} a_{x} a_{y}^{+} a_{y}-\frac{1}{2} V(x-y)\left(a_{x}^{+}-a_{y}^{+}\right)\left(a_{x}-a_{y}\right)$. The first three multicommutators have been calculated explicitly, and then from 3.2 the contributions to $\left\|K_{\Lambda}^{n}\right\|, n=1,2,3$, counted, enumerating the nonzero matrix elements and taking a supremum over $X \subset S$. The result is

$$
\left\|K_{\Lambda}^{1}\right\| \leqq \frac{7}{\sqrt{2}} \beta\|\varphi\| z, \frac{1}{2}\left\|K_{\Lambda}^{2}\right\| \leqq \frac{25}{2 \sqrt{2}} \beta^{2}\|\varphi\|^{2} z, \frac{1}{6}\left\|K_{\Lambda}^{3}\right\| \leqq \frac{78}{2 \sqrt{2}} \beta^{3}\|\varphi\|^{3} z .
$$


The remainder $\sum_{n=4}^{\infty} \frac{1}{n !}\left\|K_{\Lambda}^{n}\right\|$ can be estimated as in 3.10, using $\|\left[\varphi\left(x_{n}, y_{n}\right)\right.$, $\left.\left[\ldots\left[\varphi\left(x_{1}, y\right), a_{y}\right] \ldots\right]\right]\left\|\leqq 2^{n-4.5} 3\right\| \varphi\left(x_{n}, y_{n}\right)\|\ldots\| \varphi\left(x_{1}, y\right) \|$ for $n \geqq 3$ since $\left\|\left[\varphi\left(x_{3}, y_{3}\right),\left[\ldots\left[\varphi\left(x_{1}, y\right)\right]\right]\right]\right\| \leqq \frac{3}{2 \sqrt{2}}\left\|\varphi\left(x_{3}, y_{3}\right)\right\| \ldots\left\|\varphi\left(x_{1}, y\right)\right\|$.

Finally, compute: $(\mathscr{L} \varphi)(x)=\left(\beta^{-1} \log z+\frac{1}{2} \sum_{y \neq x} V(y-x)\right) a_{x}^{+} a_{x}$ and so $z^{-1} e^{-\frac{1}{2} \beta\|\varphi\|} \leqq \mathscr{L} z \leqq e^{\frac{1}{2} \beta\|\varphi\|} z^{-1}$.

Theorem 4.1. Let $f(t)=4.95 t+17.7 t^{2}+165 t^{3}+1325 t^{4}(1-7.66 t)^{-1}$. The correlation functionals of the isotropic Heisenberg model are analytic in the regions $z f(\beta\|\varphi\|)<1$ and $z^{-1} e^{\frac{1}{2} \beta\|\varphi\|} f(\beta\|\varphi\|)<1$. The critical temperature $T_{\mathbf{0}}$ satisfies $T_{\mathbf{0}} \leqq 10\|\varphi\| / k$.

\section{The Classical Lattice}

A similar calculation can be carried out for the classical lattice: $\varphi^{n}(X)=V(X) a^{+}(X) a(X)$. Here $\varrho(X, Y)=0$ unless $X=Y$, and the commutators can all be explicitly evaluated. $\left[\varphi\left(X_{n}\right), \ldots,\left[\varphi\left(X_{1}\right), y\right] \ldots\right]$ $=a^{+}\left(X_{n} \cup \ldots \cup X_{1}-y\right) a\left(X_{n} \cup \ldots \cup X_{1}-y\right) a_{y}$ if $y \in X_{n} \cap \ldots \cap X_{1}$, zero otherwise. There obviously is precisely one non-zero contribution to $\left\|K_{\Lambda}^{n}\right\|$ in the sum over $P, R$, and $V$ (Theorem 3.2), and so $\left\|K_{\Lambda}^{n}\right\| \leqq \prod_{i=1}^{n}$ - $\sum_{\substack{Y_{i} \subset \Lambda \\ y \notin Y_{i}}}\left|V\left(Y_{i} \cup y\right)\right|$. Finally, $\mathscr{L} z \leqq z^{-1} e^{\beta\|\varphi\|}$.

Theorem 5.1. The correlation functionals of the classical lattice are analytic in fugacity $z$ in the regions $z\left(e^{\beta|| \varphi||}-1\right)<1$ and $z^{-1} e^{\beta\|\varphi\|}\left(e^{\beta\|\varphi\|}-1\right)$ $<1$. A bound on the critical temperature is given by $T_{0} \leqq 1.8\|\varphi\| / k$, or for nearest neighbor interaction in v-dimensions, $T_{0} \leqq 3.6 v E / k$.

\section{Summary}

The region of analyticity of the correlation functionals for a two-body quantum potential is graphed in Fig. 1, along with the larger region of analyticity for the isotropic Heisenberg model. By a different method [1], Gallavotti, Miracle-Sole, and Robinson have predicted $T_{0}<10,000\|\varphi\| / k$ for the Heisenberg model, while our result is $T_{0}<10\|\varphi\| / k$. This compares to the non-rigorous critical temperature estimate of $T_{0} \approx 0.7\|\varphi\| / k$ calculated by Dombs, see [8].

For the classical lattice, the region of analyticity is also an enlargement of the region found by the same authors [1, 2] (Fig. 2). Their result was $2 z e^{\beta\|\varphi\|}\left\{\exp \left(e^{\beta\|\varphi\|}-1\right\}<1\right.$ (plus the extended region holeparticle symmetry), obtained in a manifestly classical manner by estimating the kernels of the classical Kirwood-Salzburg equation. Actually, the estimates in $[1,2]$ can be improved by noticing that (for,e.g., 


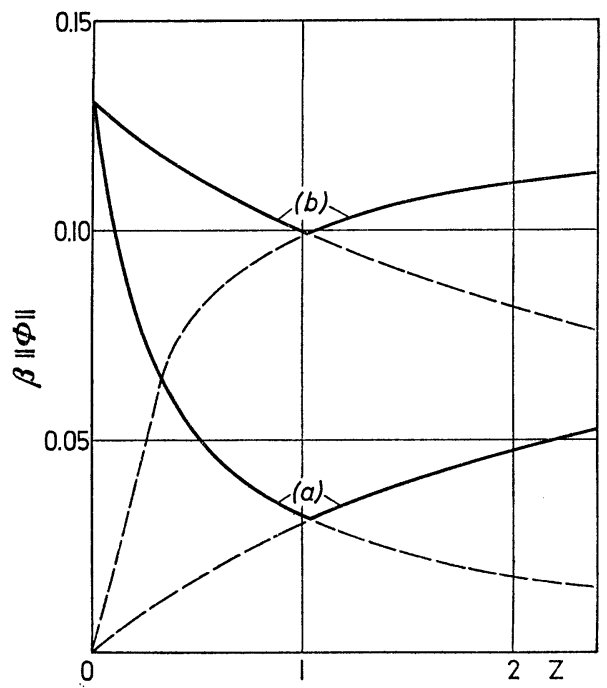

Fig. 1. The region of analyticity for the correlation functionals with: (a) a twobody quantum potential; (b) the Heisenberg model

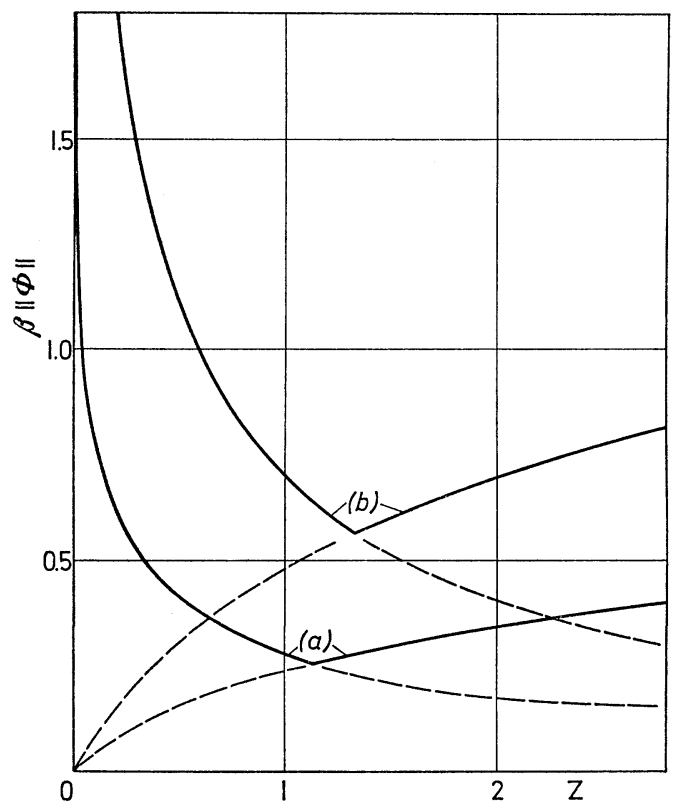

Fig. 2. The region of analyticity for the correlation functionals of a classical lattice: (a) as obtained by Gallavotit, Miracle-Sole, and Robinson [3]; (b) according to Theorem 5.1 
a two body potential) the bound on the kernels is

$$
\begin{gathered}
\sum_{R \neq \emptyset} \sum_{r \in R}\left(e^{-\varphi(r, y)}-1\right) \leqq \sum_{R \neq \emptyset} \prod_{r \in R} \sum_{n=1}^{\infty}|\varphi(r, y)|^{n} / n !=\sum_{N=1}^{\infty} \sum_{\substack{r_{1}, \ldots, r_{N} \in \mathbb{Z}^{v_{n}} \\
r_{1}<\ldots, \ldots, n_{N}}} \sum^{\infty} \\
\cdot\left|\varphi\left(r_{1}, y\right)\right|^{n_{1}} \ldots\left|\varphi\left(r_{N}, y\right)\right|^{n_{N}} / n_{1} ! \ldots n_{N} !
\end{gathered}
$$

which is the sum of all monomials of all degrees in all $\varphi(r, y)$ (weighted by $n_{r}$ !). Summing up by order, this is just

$$
\begin{gathered}
\sum_{N=1}^{\infty} \sum_{\substack{r_{1}, \ldots, r_{N} \leq Z_{v} \\
r_{1}<\ldots<r_{N}, \ldots, n_{N}=0}}|\varphi(r, y)|^{n_{1}} \ldots\left|\varphi\left(r_{N}, y\right)\right|^{n_{N}} / n_{1} ! \ldots n_{N} !=n_{N=1} \prod_{i=1}^{N} \\
\cdot\left(\sum_{r \neq y}^{N}|\varphi(r, y)|\right) \mid N !=e^{|| \varphi||}-1 .
\end{gathered}
$$

Our method predicts a critical temperature bound for classical lattices of $T_{0}<1.8\|\varphi\| / k$. The critical temperature of the Ising lattice (nearest neighbor interaction) has been calculated rigorously in two dimensions: $T_{0}=0.14\|\varphi\| / k$. Mean field theory predicts for the Ising lattice in arbitrary dimension [9]. $T_{0}<0.25\|\varphi\| / k$.

Acknowledgement. The author wishes to thank D. RoBINson for his suggestions, and A. J J $\mathrm{AFFE}$ for many helpful discussions.

\title{
References
}

1. Gallavotti, G., S. Mtracle-Sole, and D. W. Robinson: CERN reprint Th 904 (1968).

2. - Phys. Letters 25 A, 493 (1967).

3. Greenberg, W.: Commun. Math. Phys., 11, 314 (1969).

4. Gallavotti, G., and S. Mrracle-Sole: Commun. Math. Phys. 7, 274 (1968).

5. Ginibre, J.: NYU preprint (1968).

6. Whittaker, E. T., and Watson: Modern analysis, p. 253. London: Cambridge University Press 1963.

7. Griffiths, R. B.: J. Math. Phys. 5, 1215 (1964).

8. Frsher, M. E.: Repts. Prog. Phys. 30, 615 (1967).

9. Fisher, M. E., and GadNT: Phys. Rev. 133A, 224 (1964).

\author{
W. Greenberg \\ Department of Mathematics \\ Indiana University \\ Bloomington, Indiana, USA
}

